Chapter 4

\title{
WHY DOES A METAL-SUPERCONDUCTOR JUNCTION HAVE A RESISTANCE?
}

\author{
C. W. J. Beenakker
}

Instituut-Lorentz, Universiteit Leiden

P.O. Box 9506, 2300 RA Leiden, The Netherlands

\begin{abstract}
The phenomenon of Andreev reflection is introduced as the electronic analogue of optical phase-conjugation. In the optical problem, a disordered medium backed by a phase-conjugating mirror can become completely transparent. Yet, a disordered metal connected to a superconductor has the same resistance as in the normal state. The resolution of this paradox teaches us a fundamental difference between phase conjugation of light and electrons.
\end{abstract}

\section{INTRODUCTION}

In the late sixties, Kulik used the mechanism of Andreev reflection [1] to explain how a metal can carry a dissipationless current between two superconductors over arbitrarily long length scales, provided the temperature is low enough [2]. One can say that the normal metal has become superconducting because of the proximity to a superconductor. This proximity effect exists even if the electrons in the normal metal have no interaction. At zero temperature the maximum supercurrent that the metal can carry decays only algebraically with the separation between the superconductors - rather than exponentially, as it does at higher temperatures.

The recent revival of interest in the proximity effect has produced a deeper understanding into how the proximity-induced superconductivity of non-interacting electrons differs from true superconductivity of electrons having a pairing interaction. Clearly, the proximity effect does not require two superconductors. One should be enough. Consider a junc- 
tion between a normal metal and a superconductor (an NS junction). Let the temperature be zero. What is the resistance of this junction? One might guess that it should be smaller than in the normal state, perhaps even zero. Isn't that what the proximity effect is all about?

The answer to this question has been in the literature since 1979 [3], but it has been appreciated only in the last few years. A recent review [4] gives a comprehensive discussion within the framework of the semiclassical theory of superconductivity. A different approach, using random-matrix theory, was reviewed by the author [5]. In this lecture we take a more pedestrian route, using the analogy between Andreev reflection and optical phase-conjugation [6,7] to answer the question: Why does an NS junction have a resistance?

\section{ANDREEV REFLECTION AND OPTICAL PHASE-CONJUGATION}

It was first noted by Andreev in 1963 [1] that an electron is reflected from a superconductor in an unusual way. The differences between normal reflection and Andreev reflection are illustrated in Fig. 4.1. Let us discuss them separately.

- Charge is conserved in normal reflection but not in Andreev reflection. The reflected particle (the hole) has the opposite charge as the incident particle (the electron). This is not a violation of a fundamental conservation law. The missing charge of $2 e$ is absorbed into the superconducting ground state as a Cooper pair. It is missing only with respect to the excitations.
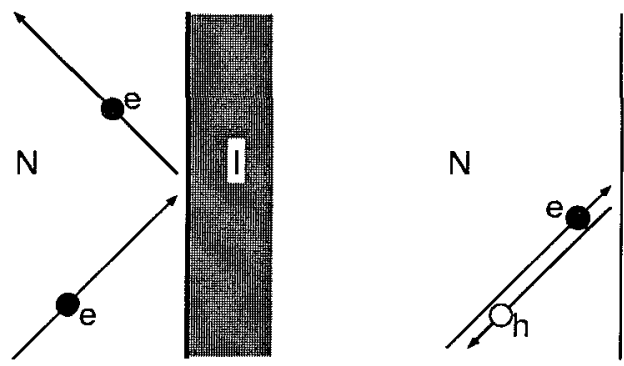

S

Figure 4.1 Normal reflection by an insulator (I) versus Andreev reflection by a superconductor (S) of an electron excitation in a normal metal (N) near the Fermi level. Normal reflection (left) conserves charge but does not conserve momentum. Andreev reflection (right) conserves momentum but does not conserve charge: The electron (e) is reflected as a hole ( $h$ ) with the same momentum and opposite velocity. The missing charge of $2 e$ is absorbed as a Cooper pair by the superconducting condensate. 
- Momentum is conserved in Andreev reflection but not in normal reflection. The conservation of momentum is an approximation, valid if the superconducting excitation gap $\Delta$ is much smaller than the Fermi energy $E_{\mathrm{F}}$ of the normal metal. The explanation for the momentum conservation is that the superconductor can not exert a significant force on the incident electron, because $\Delta$ is too small compared to the kinetic energy $E_{\mathrm{F}}$ of the electron [8]. Still, the superconductor has to reflect the electron somehow, because there are no excited states within a range $\Delta$ from the Fermi level. It is the unmovable rock meeting the irresistible object. Faced with the challenge of having to reflect a particle without changing its momentum, the superconductor finds a way out by transforming the electron into a particle whose velocity is opposite to its momentum: a hole.

- Energy is conserved in both normal and Andreev reflection. The electron is at an energy $\varepsilon$ above the Fermi level and the hole is at an energy $\varepsilon$ below it. Both particles have the same excitation energy $\varepsilon$. Andreev reflection is therefore an elastic scattering process.

- Spin is conserved in both normal and Andreev reflection. To conserve spin, the hole should have the opposite spin as the electron. This spin-flip can be ignored if the scattering properties of the normal metal are spin-independent.

The NS junction has an optical analogue known as a phase-conjugating mirror [9]. Phase conjugation is the effect that an incoming wave $\propto$ $\cos (k x-\omega t)$ is reflected as a wave $\propto \cos (-k x-\omega t)$, with opposite sign of the phase $k x$. Since $\cos (-k x-\omega t)=\cos (k x+\omega t)$, this is equivalent to reversing the sign of the time $t$, so that phase conjugation is sometimes called a time-reversal operation. The reflected wave has a wavevector precisely opposite to that of the incoming wave, and therefore propagates back along the incoming path. This is called retro-reflection. Phase conjugation of light was discovered in 1970 by Woerdman and by Stepanov, Ivakin, and Rubanov [10, 11].

A phase-conjugating mirror for light (see Fig. 4.2) consists of a cell containing a liquid or crystal with a large nonlinear susceptibility. The cell is pumped by two counter-propagating beams at frequency $\omega_{0}$. A third beam is incident with a much smaller amplitude and a slightly different frequency $\omega_{0}+\delta \omega$. The non-linear susceptibility leads to an amplification of the incident beam, which is transmitted through the cell, and to the generation of a fourth beam, which is reflected. This non-linear optical process is called "four-wave mixing". Two photons of 


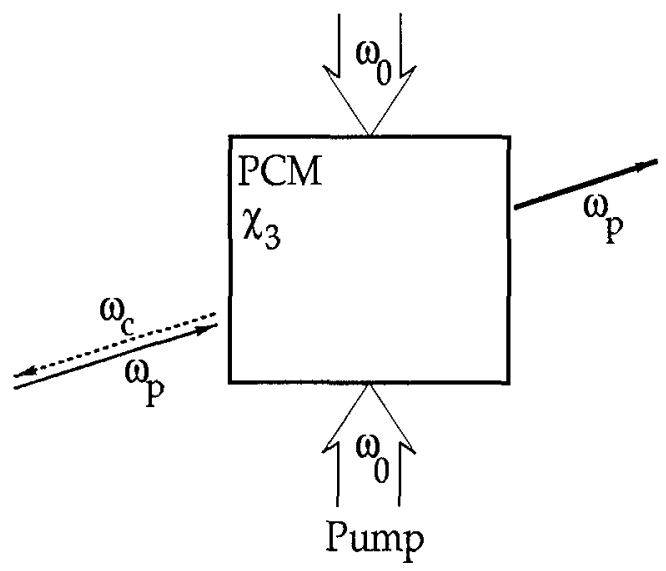

Figure 4.2 Schematic drawing of optical phase-conjugation by means of four-wave mixing. The phaseconjugating mirror (PCM) consists of a cell filled by a medium with a third-order non-linear susceptibility $\chi_{3}$. (Examples are $\mathrm{BaTiO}_{3}$ and $\mathrm{CS}_{2}$.) The medium is pumped by two counter-propagating beams at frequency $\omega_{0}$. A probe beam incident at frequency $\omega_{\mathrm{p}}=\omega_{0}+\delta \omega$ is then retro-reflected as a conjugate beam at frequency $\omega_{c}=\omega_{0}-\delta \omega$. From Ref. [12].

the pump beams are converted into one photon for the transmitted bearn and one for the reflected beam. Energy conservation dictates that the reflected beam has frequency $\omega_{0}-\delta \omega$. Momentum conservation dictates that its wavevector is opposite to that of the incident beam. Comparing retro-reflection of light with Andreev reflection of electrons, we see that the Fermi energy $E_{\mathrm{F}}$ plays the role of the pump frequency $\omega_{0}$, while the excitation energy $\varepsilon$ corresponds to the frequency shift $\delta \omega$.

A phase-conjugating mirror can be used for wavefront reconstruction. Imagine an incoming plane wave, that is distorted by some inhomogeneity. When this distorted wave falls on the mirror, it is phase conjugated and retro-reflected. Due to the time-reversal effect, the inhomogeneity that had distorted the wave now changes it back to the original plane wave. An example is shown in Fig. 4.3. Complete wavefront reconstruction is possible only if the distorted wavefront remains approximately planar, since perfect time reversal upon reflection holds only in a narrow range of angles of incidence for realistic systems. This is an important, but not essential complication, that we will ignore in what follows.

\section{THE RESISTANCE PARADOX}

We have learned that a disordered medium (such as the frosted glass in Fig. 4.3) becomes transparent when it is backed by a phase-conjugating mirror. By analogy, one would expect that a disordered metal backed by a superconductor would become "transparent" too, meaning that its resistance should vanish (up to a small contact resistance that is present even without any disorder). This does not happen. Upon decreasing the temperature below the superconducting transition temperature, the resistance drops slightly but then rises again back to its high-temperature value. (A recent experiment is shown in Fig. 4.4, where the conduc- 

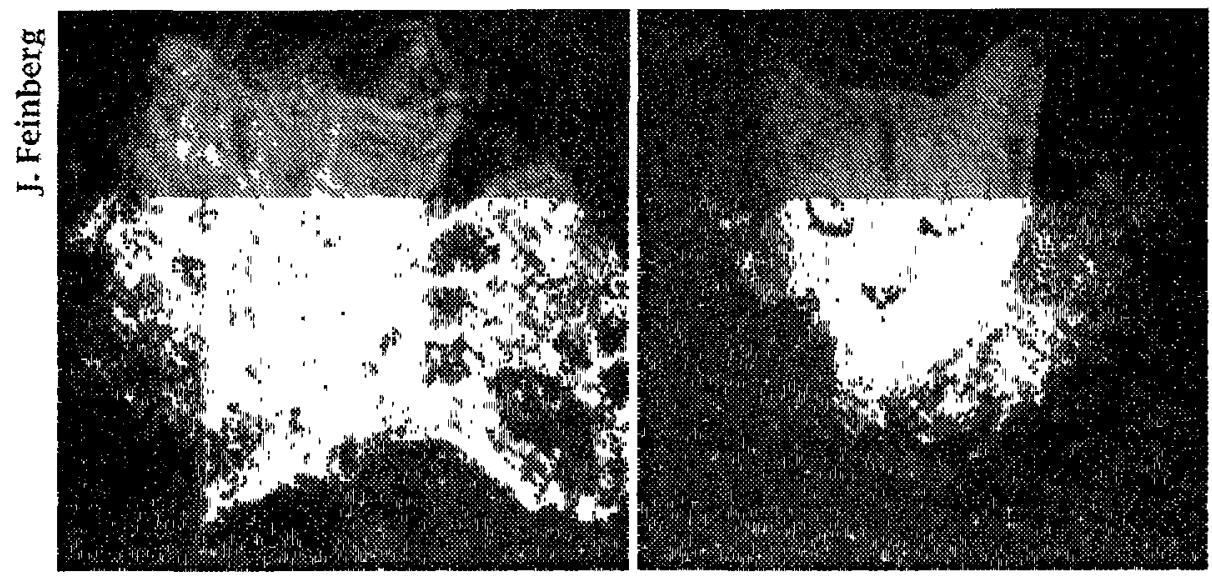

Figure 4.3 Example of wavefront reconstruction by optical phase-conjugation. In both photographs the image of a cat was distorted by transmitting it through a piece of frosted glass, and reflecting it back through the same piece of glass. This gives an unrecognizable image when reflected by an ordinary mirror (left panel) and the original image when reflected by a phase-conjugating mirror (right panel). From Ref. [13].

tance is plotted instead of the resistance.) This so-called "re-entrance effect" has been reviewed recently by Courtois et al. [4], and we refer to
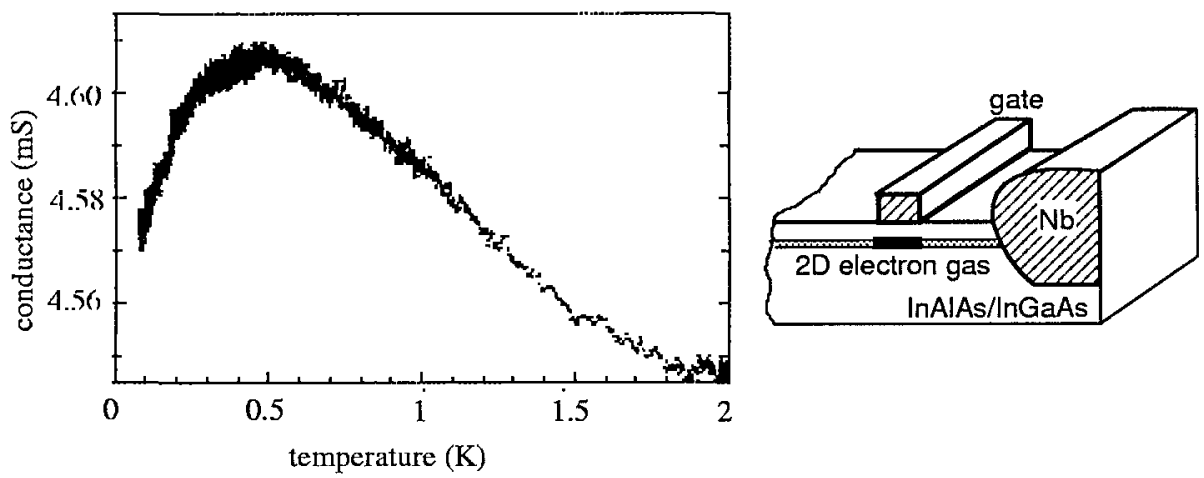

Figure 4.4 Temperature dependence of the conductance of an NS junction, showing the re-entrance effect. The superconductor is $\mathrm{Nb}$, the normal metal is a twodimensional electron gas. A gate creates a strongly disordered region in the $2 \mathrm{D}$ gas that dominates the conductance of the junction. Upon lowering the temperature the conductance first rises and then drops again. Under ideal circumstances the low- and high-temperature limits would be the same. From Ref. [16]. 
that review for an extensive list of references. The theoretical prediction $[3,14,15]$ is that at zero temperature the resistance of the normal-metalsuperconductor junction is the same as in the normal state. How can we reconcile this with the notion of Andreev reflection as a "time-reversing" process, analogous to optical phase-conjugation? To resolve this paradox, let us study the analogy more carefully, to see where it breaks down.

For a simple discussion it is convenient to replace the disordered medium by a tunnel barrier (or semi-transparent mirror) and consider the phase shift accumulated by an electron (or light wave) that bounces back and forth between the barrier and the superconductor (or phaseconjugating mirror). A periodic orbit (see Fig. 4.5) consists of two roundtrips, one as an electron (or light at frequency $\omega_{0}+\delta \omega$ ), the other as a hole (or light at frequency $\omega_{0}-\delta \omega$ ). The miracle of phase conjugation is that phase shifts accumulated in the first round trip are cancelled in the second round trip. If this were the whole story, one would conclude that the net phase increment is zero, so all periodic orbits would interfere constructively and the tunnel barrier would become transparent because of resonant tunneling.

But it is not the whole story. There is an extra phase shift of $-\pi / 2$ acquired upon Andreev reflection that destroys the resonance. Since the periodic orbit consists of two Andreev reflections, one from electron to hole and one from hole to electron, and both reflections have the same phase shift $-\pi / 2$, the net phase increment of the periodic orbit is $-\pi$ and not zero. So subsequent periodic orbits interfere destructively, rather than constructively, and tunneling becomes suppressed rather than enhanced. In contrast, a phase-conjugating mirror adds a phase shift that alternates between $+\pi / 2$ and $-\pi / 2$ from one reflection to the next, so the net phase increment of a periodic orbit remains zero.

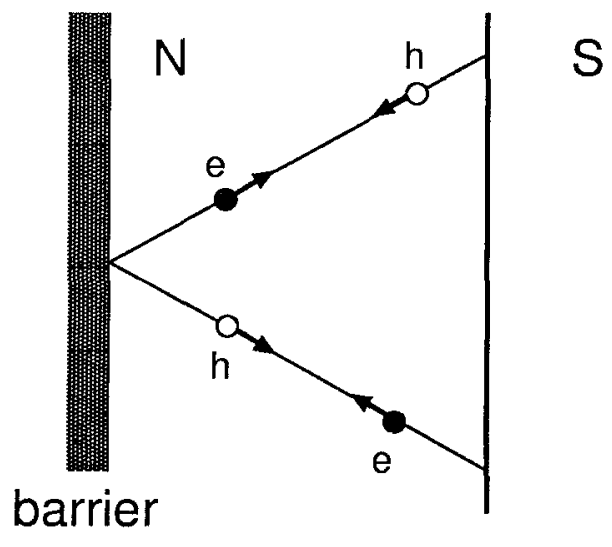

Figure 4.5 Periodic orbit consisting of two normal reflections and two retro-reflections. The net phase increment is zero in the optical case and $-\pi$ in the electronic case. Hence the periodic orbits interfere constructively for light and destructively for electrons. This explains why the barrier becomes transparent for light but not for electrons. 
For a more quantitative description of the conductance we need to compute the probability $R_{\text {he }}$ that an incident electron is reflected as a hole. The matrix of probability amplitudes $r_{\text {he }}$ can be constructed as a geometric series of multiple reflections:

$$
\begin{aligned}
r_{\text {he }} & =t^{\dagger} \frac{1}{\mathrm{i}} t+t^{\dagger} \frac{1}{\mathrm{i}} r \frac{1}{\mathrm{i}} r^{\dagger} \frac{1}{\mathrm{i}} t+t^{\dagger} \frac{1}{\mathrm{i}}\left[r \frac{1}{\mathrm{i}} r^{\dagger} \frac{1}{\mathrm{i}}\right]^{2} t+\cdots \\
& =t^{\dagger} \frac{1}{\mathrm{i}}\left[1-r \frac{1}{\mathrm{i}} r^{\dagger} \frac{1}{\mathrm{i}}\right]^{-1} t .
\end{aligned}
$$

Each factor $1 / \mathrm{i}=\exp (-\mathrm{i} \pi / 2)$ corresponds to an Andreev reflection. The matrices $t, t^{\dagger}$ and $r, r^{\dagger}$ are the $N \times N$ transmission and reflection matrices of the tunnel barrier, or more generally, of the disordered region in the normal metal. (The number $N$ is related to the cross-sectional area $A$ of the junction and the Fermi wavelength $\lambda_{\mathrm{F}}$ by $N \simeq A / \lambda_{\mathrm{F}}^{2}$.) The matrices $t, r$ pertain to the electron and the matrices $t^{\dagger}, r^{\dagger}$ to the hole. The resulting reflection probability $R_{\mathrm{he}}=N^{-1} \operatorname{Tr} r_{\mathrm{he}} r_{\mathrm{he}}^{\dagger}$ is given by [14]

$$
R_{\mathrm{he}}=\frac{1}{N} \operatorname{Tr}\left(\frac{t t^{\dagger}}{1+r r^{\dagger}}\right)^{2}=\frac{1}{N} \operatorname{Tr}\left(\frac{t t^{\dagger}}{2-t t^{\dagger}}\right)^{2} .
$$

We have used the relationship $t t^{\dagger}+r r^{\dagger}=1$, dictated by current conservation. The conductance $G_{\mathrm{NS}}$ of the NS junction is related to $R_{\mathrm{he}}$ by $[17,18]$

$$
G_{\mathrm{NS}}=\frac{4 e^{2}}{h} N R_{\mathrm{he}}
$$

In the optical analogue one has the probability $R_{ \pm}$for an incident light wave with frequency $\omega_{0}+\delta \omega$ to be reflected into a wave with frequency $\omega_{0}-\delta \omega$. The matrix of probability amplitudes is given by the geometric series

$$
\begin{aligned}
r_{ \pm} & =t^{\dagger} \frac{1}{\mathrm{i}} t+t^{\dagger} \frac{1}{\mathrm{i}} r \mathrm{i} r^{\dagger} \frac{1}{\mathrm{i}} t+t^{\dagger} \frac{1}{\mathrm{i}}\left[r \mathrm{i} r^{\dagger} \frac{1}{\mathrm{i}}\right]^{2} t+\cdots \\
& =t^{\dagger} \frac{1}{\mathrm{i}}\left[1-r \mathrm{i} r^{\dagger} \frac{1}{\mathrm{i}}\right]^{-1} t .
\end{aligned}
$$

The only difference with Eq. (4.1) is the alternation of factors $1 / \mathrm{i}$ and $i$, corresponding to the different phase shifts $\exp ( \pm \mathrm{i} \pi / 2)$ acquired at the phase-conjugating mirror. The reflection probability $R_{ \pm}=N^{-1} \operatorname{Tr} r_{ \pm} r_{ \pm}^{\dagger}$ now becomes independent of the disorder [19],

$$
R_{ \pm}=\frac{1}{N} \operatorname{Tr}\left(\frac{t t^{\dagger}}{1-r r^{\dagger}}\right)^{2}=1
$$


The disordered medium has become completely transparent.

It is remarkable that a small difference in phase shifts has such far reaching consequences. Note that one needs to consider multiple reflections in order to see the difference: The first term in the series is the same in Eqs. (4.1) and (4.4). That is probably why this essential difference between Andreev reflection and optical phase-conjugation was not noticed prior to Ref. [19].

\section{HOW BIG IS THE RESISTANCE?}

Now that we understand why a disordered piece of metal connected to a superconductor does not become transparent, we would like to go one step further and ask whether the resistance (or conductance) is bigger or smaller than without the superconductor. To that end we compare, following Ref. [14], the expression for the conductance of the NS junction [obtained from Eqs. (4.2) and (4.3)],

$$
G_{\mathrm{NS}}=\frac{4 e^{2}}{h} \sum_{n=1}^{N} \frac{T_{n}^{2}}{\left(2-T_{n}\right)^{2}},
$$

with the Landauer formula for the normal-state conductance,

$$
G_{\mathrm{N}}=\frac{2 e^{2}}{h} \sum_{n=1}^{N} T_{n}
$$

The numbers $T_{1}, T_{2}, \ldots T_{N}$ are the eigenvalues of the matrix product $t t^{\dagger}$. These transmission eigenvalues are real numbers between 0 and 1 that depend only on the properties of the metal (regardless of the superconductor). Both formulas (4.6) and (4.7) hold at zero temperature, so we will be comparing the zero-temperature limits of $G_{\mathrm{NS}}$ and $G_{\mathrm{N}}$.

Since $x^{2} /(2-x)^{2} \leq x$ for $x \in[0,1]$, we can immediately conclude that $G_{\mathrm{NS}} \leq 2 G_{\mathrm{N}}$. If there is no disorder, then all $T_{n}$ 's are equal to unity, hence $G_{N S}$ reaches its maximum value of $2 G_{N}$. For a tunnel barrier all $T_{n}$ 's are $\ll 1$, hence $G_{\mathrm{NS}}$ drops far below $G_{\mathrm{N}}$. A disordered metal will lie somewhere in between these two extremes, but where?

We have already alluded to the answer in the previous section, that $G_{\mathrm{NS}}=G_{\mathrm{N}}$ for a disordered metal in the zero-temperature limit. To derive this remarkable equality, we parameterize the transmission eigenvalue $T_{n}$ in terms of the localization length $\zeta_{n}$,

$$
T_{n}=\frac{1}{\cosh ^{2}\left(L / \zeta_{n}\right)}
$$


where $L$ is the length of the disordered region. Substitution into Eqs. (4.6) and (4.7) gives the average conductances

$$
\begin{aligned}
\left\langle G_{\mathrm{NS}}\right\rangle_{L} & =\frac{4 e^{2}}{h} N \int_{0}^{\infty} d \zeta P_{L}(\zeta) \cosh ^{-2}(2 L / \zeta) \\
\left\langle G_{\mathrm{N}}\right\rangle_{L} & =\frac{2 e^{2}}{h} N \int_{0}^{\infty} d \zeta P_{L}(\zeta) \cosh ^{-2}(L / \zeta)
\end{aligned}
$$

(For Eq. (4.9) we have used that $2 \cosh ^{2} x-1=\cosh 2 x$.) The probability distribution $P_{L}(\zeta)$ of $\zeta$ is independent of $L$ in a range of lengths between $l$ and $N l[5]$. It then follows immediately that

$$
\left\langle G_{\mathrm{NS}}\right\rangle_{L}=2\left\langle G_{\mathrm{N}}\right\rangle_{2 L}
$$

Since $G_{\mathrm{N}} \propto 1 / L$, according to Ohm's law, we arrive at the equality of $G_{\mathrm{NS}}$ and $G_{\mathrm{N}}$.

The restriction to the range $l \ll L \ll N l$ is the restriction to the regime of diffusive transport: For smaller $L$ we enter the ballistic regime and $G_{\mathrm{NS}}$ rises to $2 G_{N}$; For larger $L$ we enter the localized regime, where tunneling takes over from diffusion and $G_{\mathrm{NS}}$ becomes $\ll G_{\mathrm{N}}$.

\section{CONCLUSION}

We have learned a fundamental difference between Andreev reflection of electrons and phase-conjugation of light. While it is appealing to think of the Andreev reflected hole as the time reverse of the incident electron, this picture breaks down upon closer inspection. The phase shift of $-\pi / 2$ acquired upon Andreev reflection spoils the time-reversing properties and explains why a disordered metal does not become transparent when connected to a superconductor.

\section{Acknowledgements}

The research on which this lecture is based was done in collaboration with J. C. J. Paasschens. It was supported by the "Stichting voor Fundamenteel Onderzoek der Materie" (FOM) and by the "Nederlandse organisatie voor Wetenschappelijk Onderzoek" (NWO).

\section{References}

[1] A. F. Andreev, Zh. Eksp. Teor. Fiz. 46, 1823 (1964) [Sov. Phys. JETP 19, 1228 (1964)].

[2] I. O. Kulik, Zh. Eksp. Teor. Fiz. 57, 1745 (1969) [Sov. Phys. JETP 30, 944 (1970)].

[3] S. N. Artemenko, A. F. Volkov, and A. V. Zaŭtsev, Solid State Comm. 30, 771 (1979). 
[4] H. Courtois, P. Charlat, Ph. Gandit, D. Mailly, and B. Pannetier, J. Low Temp. Phys. 116, 187 (1999).

[5] C. W. J. Beenakker, Rev. Mod. Phys. 69, 731 (1997).

[6] D. Lenstra, in: Huygens Principle 1690-1990; Theory and Applications, edited by H. Blok, H. A. Ferweda, and H. K. Kuiken (NorthHolland, Amsterdam, 1990).

[7] H. van Houten and C. W. J. Beenakker, Physica B 175, 187 (1991).

[8] A. A. Abrikosov, Fundamentals of the Theory of Metals (NorthHolland, Amsterdam, 1988).

[9] D. M. Pepper, Sci. Am. 254 (1), 56 (1986).

[10] J. P. Woerdman, Optics Comm. 2, 212 (1970).

[11] B. I. Stepanov, E. V. Ivakin, and A. S. Rubanov, Dokl. Akad. Nauk USSR 196, 567 (1971) [Sov. Phys. Dokl. 16, 46 (1971)].

[12] J. C. J. Paasschens, Ph.D. Thesis (Universiteit Leiden, 1997).

[13] J. Feinberg, Opt. Lett. 7, 486 (1982).

[14] C. W. J. Beenakker, Phys. Rev. B 46, 12841 (1992).

[15] Yu. V. Nazarov and T. H. Stoof, Phys. Rev. Lett. 76, 823 (1996).

[16] E. Toyoda, H. Takayanagi, and H. Nakano, Phys. Rev. B 59, 11653 (1999).

[17] G. E. Blonder, M. Tinkham, and T. M. Klapwijk, Phys. Rev. B 25, 4515 (1982).

[18] Y. Takane and H. Ebisawa, J. Phys. Soc. Japan 61, 3466 (1992).

[19] J. C. J. Paasschens, M. J. M. de Jong, P. W. Brouwer, and C. W. J. Beenakker, Phys. Rev. A 56, 4216 (1997). 